\title{
sciendo
}

\section{Improvements in PD models. A case-study approach}

\author{
Raluca Dana CAPLESCU \\ Bucharest University of Economic Studies, Bucharest, Romania \\ raluca.caplescu@csie.ase.ro \\ Manuela-Simona COJOCEA \\ University of Bucharest, Bucharest, Romania \\ simona.cojocea@fmi.unibuc.ro \\ Daniel Traian PELE \\ Bucharest University of Economic Studies, Bucharest, Romania \\ danpele@ase.ro \\ Vasile Alecsandru STRAT \\ Bucharest University of Economic Studies, Bucharest, Romania \\ vasile.strat@bbs.ase.ro
}

\begin{abstract}
Models for estimating the probability of default are widely used in the business throughout the lending process, starting as early as the application stage, where they play an important role in loan approval status. For model soundness and performance, ensuring adequate data quality is essential. Identifying outliers, analyzing their impact and choosing the right method to treat them is a necessary stage of preprocessing, which is often overlooked in practice for a variety of reasons, an important one being insufficient data. Given the inherent imbalance of the loan portfolio with regard to default status, elimination of outliers is seldom feasible. The current widely accepted approach is based on binning and weight of evidence. Usually two types of binning are tested, namely bucket and quantile. While the latter is robust to outlier presence, the former is not. Both approaches lead to the discretization of the continuous variable they are applied on. This causes information loss both in terms of variation given by individual values and in terms of distance between the various observation points on a certain variable. In the present paper, we explore the opportunity of using other methods for dealing with outlier presence and we describe their advantages and disadvantages in the context of probability of default estimation for credit risk. We conclude that, aside from quantile binning, not dealing with outliers in case of very large datasets or winsorizing are also effective. More importantly, several methods should be considered and tested for each variable in order to find the optimal balance between altering the data and reducing variance.
\end{abstract}

Keywords: PD models, outlier treatment, impact analysis, P2P lending, Lending Club.

\section{Introduction}

Lending money for cost has always been a profitable business, provided that the customers act in good faith and pay the monthly due amount. If the number of customers is relatively low and only a small proportion of them fail to pay the installments, the lender can track those customers and use all the legal ways at their disposal to retrieve the amounts they borrowed. But if the number of customers is high and the outstanding amount is relatively small, taking any form of legal action against a non-paying customer might not prove cost-effective. In this case, the creditor will settle for the loss, instead of investing time and other resources trying to track down the faulty loan and collect the money. 
To limit the loss generated by non-performing credit loans (Pop et al.), a serious scrutiny of the applications is necessary. This step implies a cost with the human resource, especially when the number of applications is very high. Ideally, the full automation of the loan application ranking process would speed up the entire procedure, eliminating the bias introduced by the human factor, but as no algorithm is perfect, this would leave the financial institution vulnerable to other kinds of risks, such as diminishing the business volume by mistakenly rejecting too many loan applications or conversely, adding in their portfolio too many high risk loans, thus increasing the costs.

In practice, a hybrid approach (Hadad and Bratianu, 2019) is optimal: the application firstly goes through an automatic evaluation, followed by the final decision being made by a credit officer. This way, the procedure is sped up and the human resource is only used to make a judgment call where the algorithmic approach is not relevant. For this reason, building a good and reliable classification model is of great interest for financial institutions and consequently a lot of literature and statistical studies focused on precisely this goal.

As technology evolves, borrowing activity diversifies as peer-to-peer (P2P) lending platforms become more popular and this creates alternative funding opportunities for those who need it. With terms and conditions favoring the customer more, P2P lending is changing the traditional business model used by the banks. The online platforms which facilitate $\mathrm{P} 2 \mathrm{P}$ lending make the acceptance or rejection of a credit loan application more transparent and allow a loan to be partially funded by several lenders depending on their risk aversion.

To be competitive while running a good business, P2P lending is creating an even higher demand for very efficient classification algorithms than the banks did before. Distinguishing between good and bad clients becomes vital to lower the default risk, and that puts data specialists on the spotlight as not only do they need to create better models, but they also need to pay more attention to the way the data is being collected and preprocessed, as this could have an important impact on the final result.

Traditionally, the data cleaning process is looking to eliminate incomplete and incorrect data before creating a model based on it. Human error and faulty measurement tools can mistakenly allow very large or extremely small values to be collected and processed as "good data". This can have a great impact on the statistical analysis of the data, which can be severely misled by these extreme values called outliers. While there's no rigorous mathematical definition, an intuitive approach would present an outlier to be "an observation which deviates so much from other observations as to arise suspicions that it was generated by a different mechanism" (Hawkins, 1980, p. 1).

If the outliers are nothing but mistakenly introduced values, eliminating them from the data is the correct course of action, but that is not always the case. There are times when extreme values are in fact valid, but they look suspicious because of the reduced size of the sample or simply because they represent uncommon, while still possible, values for a particular variable. For example, if most applicants for a credit loan have their income in a certain range, finding an application in which the customer has an income considerably larger than the superior margin of this range raises some suspicions.

As Hawkins (1980) states, there are two mechanisms resulting in the genesis of outliers. The first one appears when data come from a heavy tailed distribution such as the Student distribution. Formalizing this model, two classes of probability distribution can be identified (Green, 1976): outlier-prone and outlier-resistant. The outlier-prone family of distributions has tails which go to 0 slowly, while the outlier-resistant family has very thin tails (for example, the normal 
distribution and the gamma family of distributions). The second mechanism generating outliers appears when the data come from two distributions: the first one, the basic distribution, generates the "good" observations and the second one, the contaminating distribution, generates the "contaminants". If the second distribution has heavier tails than the basic distribution, then the "contaminants" will tend to visibly separate from the "good values" and become outliers.

When outliers arise through the second mechanism it is important to identify and eliminate them from the data, as they do not hold any useful information about the "good distribution". But when outliers are generated by the means of the first mechanism, removing them will create a distorted image of the phenomenon revealed by the data. Not having information regarding which mechanism generated the outliers, we should be cautious about removing them completely from the data and consider replacing them with appropriate values instead. This is relevant especially when the sample size is small, and we cannot afford to eliminate the outliers. That is often the case with imbalanced data, as the number of clients who defaulted in the past is significantly smaller than the number of those who did not.

The paper will continue with the literature review section, where we present current knowledge on outlier detection, impact and treatment in the context of probability of default modelling, followed by presenting the data used, preprocessing performed and model development. The methodology section also details the outlier treatment approaches and evaluation metrics used for comparisons. We then present and compare the results, limitations and potential future research directions and conclude the paper.

\section{Literature review}

The range of problems gravitating around the topic of outliers is vast because they are at the core of many misleading estimations. An essential step in the data cleaning process is their identification and analysis of the impact they have. From reaching consensus regarding its definition, to deciding on the best way to deal with their presence, outliers are the focus of many scientific endeavors across all fields and disciplines (Chawla et al., 2002; Nnamoko and Korkontzelos, 2020; Sharma et al., 2015; Smith and Martinez, 2011; Tallón-Ballesteros and Riquelme, 2014; Wang et al., 2021).

Once defined and identified, the question remains how to deal with the presence of outliers during the modelling stage. Simply removing them is not always feasible, especially when the data is scarce, despite opinions stated to the contrary (Sharma et al., 2015). Impact analysis in case of outliers would assess whether various approaches improve the performance of the model and choose the approach accordingly, particularly when partial elimination of outliers leads to better results and less loss of information (Tallón-Ballesteros and Riquelme, 2014).

The most frequent definition of outliers is the one based on the interquartile range (IQR) and it classifies as outliers observations that are 1.5 IQR below the first quartile or above the third one, respectively. The main advantage of this method is that is it easy to apply so its widespread across various fields and application domains, as well as for preprocessing input data for a wide range of algorithms and methods (Nnamoko and Korkontzelos, 2020; Tallón-Ballesteros and Riquelme, 2014; Wang et al., 2021). Conversely, it has the disadvantage that it assumes normal or close to normal distribution of the data.

A particularly challenging task for classification problems is handling outliers in imbalanced datasets, as if always the case for probability of default models. Among the methods proposed for dealing with the imbalance is also a technique called SMOTE (Synthetic Minority Over-sampling TEchnique) (Chawla et al., 2002). Classic under-sampling diminishes the majority class by randomly selecting from it a number of observations roughly equal to that of the minority 
class, while classic over-sampling randomly duplicates the minority class several times in order to get a balanced sample. As opposed to these approaches, SMOTE creates new artificial instances based on information from neighboring observations in the minority class.

The enhancement of outlier presence through oversampling led to the tackling of class imbalance with SMOTE. Outliers are seen as sparse "good values" and the approach is global, namely focused on the entire dataset (irrespective of class) (Chawla et al., 2002). Applying this technique exposes the learning algorithm to rarer cases, which may be difficult to learn otherwise. For this reason, outlier removal can sometimes harm model performance rather than enhance it. As the learning algorithms have a wider visibility of the sparse instances, the classification ability of the model is boosted. One caveat is to be able to identify mistakenly introduced values and eliminate them from the sample.

The importance of analyzing and treating outliers is magnified by the type of model that will be used on the resulting dataset. As a particular case of Generalized Linear Model (GLM), the logistic regression (LR) is sensible to outlier presence. But given its reduced complexity and its easy implementation, LR is the default "go to" model in classification problems and often the preferred benchmark in comparison studies. it is natural to be considered frequently as a base model in classification studies, when different models are being compared.

As opposed to other statistical models which are used to predict the values for the response variable, LR outputs the probability that the response variable falls into a certain category. This particularity gives the model great flexibility regarding an acceptable risk threshold. The latter can be obtained by conducting an empirical study and then be set manually. Having the possibility of easily modifying the risk threshold favors the development of further what-if type of analysis.

A consequence of its advantages is that LR is the most widely used classifier in probability of default estimation models. Being easily interpretable allows the lender and the regulators to understand the impact of each scoring factor and to calibrate the risk strategy accordingly. Far from being a "one size fits all" kind of model, it was proven in several studies that for probability of default estimation LR outperformed models based on Random Forest and Naïve Bayes (Abdemoula, 2015; Çığşar and Ünal, 2019), K Nearest Neighbor (KNN) (Abdemoula, 2015) and discriminant analysis or probit (Gurný and Gurný, 2013). In terms of business utility, LR models were found to be among the most accurate in several studies focused on private individuals (Akcura and Chhibber, 2018; Bayraci and Susuz, 2019; Çığşar and Ünal, 2019; Gurný and Gurný, 2013; Kaya et al., 2008; Nabende and Senfuma, 2019; Nh and Mai, 2018; Obare and Muraya, 2018; Sariev and Germano, 2020; Turlík, 2018).

\section{Methodology}

The current section will provide details regarding exploratory data analysis and data preparation performed during this stage, as well as the several approaches for identifying and treating outliers. The comparison will be done based on results of logistic regression, which we chose because it is the standard in the industry. The entire analysis was performed using Python's modules numpy, pandas and sklearn.

\section{Data processing and modelling}

The Lending Club dataset (Lending Club) is available on Kaggle for loans financed between June 2007 and December 2018. It contains information about 2.26 million loans and it has 151 variables.

For practical reasons, we will focus only on finalized loan contracts, so only fully paid and charged off records are considered for this analysis. Also, to avoid including noise in the data or 
mitigate the risk of seeing noise as significant due to very large amounts of data, we selected only the most recent three years in the sample, namely 2016-2018. The chosen sample consists of about 518 thousand observations, of which approximately $22 \%$ are charged off. As for all loan portfolios, there is class imbalance, but it's not as extreme as in the case of traditional banking.

The large number of variables imposes a feature selection step. The first thing we looked at was the usability of the variables, which we measured based on percent of missing values. Given that imputation is very difficult for variables with a high missing rate, we decided to drop all columns for which we had more than $30 \%$ missing values. Also, to make the model design as straightforward as possible, we decided to focus only on the information available to a potential lender when deciding whether to invest in the loan or not. The only variable that we kept despite not being available before investment is the date for the loan issuing, which we use for splitting the sample into training and testing sub-samples and dropping it subsequently.

Several more columns were dropped after exploratory analysis for various reasons. The unique loan identifier (id) did not contain useful information for modelling. Another category of variables contained pairs that provide similar insight, but at different levels of granularity, so in order to simplify the model the most granular was dropped (grade and subgrade, purpose and title). Some variables had too many categories so they were dropped because turning them into dummies would have made the model highly complex and they were not much correlated to the target variable either (employment title, zip code, address state). The FICO score was given as a range of values with a lower and upper bound, so, after checking that they were highly correlated, we computed the mean between the two and deleted them. We also applied a log-transformation to the variables about the borrower's annual income and revolving balance and dropped the original values. This step made their respective distributions resemble more to the normal one.

Among the remaining 27 columns, the categorical ones were converted into dummy variables. Before the conversion we checked for missing values and dropped the row containing missing values on inquiries in the past 6 months. We did that because, being a categorical variable, it would have required us to create a dummy variable for the missing category as well and since there was only one missing observation, this made no sense. The target variable was coded with 1 for charged off records and 0 for the fully paid ones. Following these steps, the final dataset has 518705 loans and 41 columns.

For testing purposes, we did a train/test split based on issue date such that $75 \%$ of the issue dates are in the training sample and the remaining in the test sample. This approach led to including the loans up until June 2017 in the training sample and the period between July 2017 and December 2018 was used for testing purposes (about $26 \%$ of the data). The charge off rate is $23.3 \%$ for training and $19.9 \%$ for tests.

Data leakage and input consistency are important aspects to consider when modelling so we created a pipeline on which we impute missing data in the continuous variables with the median, standardize the input data and estimate the model. The standardization is done using the StandardScaler in sklearn and the model is the logistic regression. For validation purposes we use a 10 -fold cross-validation (CV). 


\section{Outlier treatment}

Outlier identification and analysis is a crucial step in the modelling process. In the present study, we define as outlier a value that is outside the interval given by the following lower and upper bounds:

$$
\begin{aligned}
& \text { lower bound }=Q 1-1.5 I Q R \\
& \text { upper bound }=Q 3+1.5 I Q R
\end{aligned}
$$

PICBE |

where:

Q1 - is the first quartile (position for which $25 \%$ of the data are smaller);

Q3 - is the third quartile (position for which $25 \%$ of the data are larger);

$\mathrm{IQR}=\mathrm{Q} 3-\mathrm{Q} 1-$ is the interquartile range (which contains the middle $50 \%$ of the data).

Despite its importance and impact, outlier analysis is often overlooked or simplified. The reasons can be complex, ranging from faulty model design or lack of experience on the developer's side to insufficient data. Given the inherent imbalance of the loan portfolio with regard to default status, elimination of outliers is not always feasible. And when it is, it leads to loss of potentially valuable information. Conversely, not treating the outliers in any way can lead to expensive mistakes.

The current widely accepted approach is based on binning, two types of which are usually tested, namely bucket binning and quantile binning. The former generates intervals of the same length, but is highly sensitive to outliers since it relies on the value range. In quantile binning the number of observations is approximately equally divided between the desired number of bins. This approach is robust to outlier presence because it's based on positional indicators, rather than actual values in the data. Both methods eventually convert continuous variables into discrete ones. This leads to information loss both in terms of variation given by individual values and in terms of distance between the various observation points on a certain variable.

Another approach often considered when dealing with outliers is to replace them with the median, which, in contrast to the mean, is robust to their presence. Following this approach, the mean and median values are most often getting closer and the variation in the data can be substantially reduced, but unusual data points are treated as central, most common points. This may lead to changing patterns and relationships in the data, which also has negative impact on model performance.

A less drastic version of this method is winsorizing, which takes the principle on which replacing with the median is based and adapts it to maintain the observations in their respective distribution tails. To be more precise, for winsorizing we chose two quantiles, one for each extreme of the range and replace all values outside the interval between them with the interval boundaries. For example, a 5\% winsorizing would mean replacing all values smaller than the fifth percentile (P5) or larger than the $95^{\text {th }}$ percentile (P95) with P5 and P95, respectively. In this case decrease in variation and shifting of the mean are still observed, albeit to a lesser extent, but the data points are kept at the extremities of the range, rather than brought in the middle.

All these approaches have advantages and disadvantages. We will build a logistic regression model on each variant to see in which way would it be impacted by no treatment, elimination, binning, replacing with median and winsorizing for variables with outliers. The choice 
of the model was based on its widespread use in the banking industry, as it allows for transparency so it's preferred by most regulators.

\section{Evaluation metrics}

To evaluate model performance, it is important to choose the right tools for that purpose. Six of the most used metrics for classification, namely accuracy, balanced accuracy, F1-score, recall, precision and ROC AUC are presented briefly.

Accuracy in classification problems represents the proportion of correct predictions made by the model with respect to the total amount of predictions. Accuracy is a good measure when the target variable classes in the data are nearly balanced, but it tends to be misleading when that is not the case.

$$
\text { Accuracy }=\frac{\text { Fully paid predicted as such }+ \text { Charged off predicted as such }}{\text { Total number of loans }}
$$

Given the unbalanced nature of our sample, accuracy is not a good metric as it could lead to false conclusions. In the dataset we analyze, about one in five loans are charged off so, even if the model classified all the credits as being fully paid, the accuracy would still be $80 \%$. While this is a good level of accuracy, the model is useless since it failed to identify the category of interest.

Balanced accuracy ratio maintains the same basic idea, but it applies to each class individually. Subsequently, the average of the two results is computed to obtain a more accurate representation of the model performance. This way, the possibility of an artificially inflated accuracy is much reduced.

$$
\text { Balanced accuracy }=\frac{\frac{\text { Fully paid predicted as such }}{\text { All fully paid loans }}+\frac{\text { Charged off predicted as such }}{\text { All charged off loans }}}{2}
$$

In the example above, the balanced accuracy ratio would be the mean of $100 \%$ correctly predicted fully paid loans and $0 \%$ correctly predicted charged off loans. While much lower than the $80 \%$ obtained with accuracy, it suggests half of the loans are correctly predicted in their respective categories, which is, once again, misleading.

The goal of our study is to identify the clients who are very likely to charge off, so we are not so much interested in the overall performance of the model, but more on the good prediction of this specific class. That is why we will consider metrics which focus on the model's capacity to predict the class of interest.

Recall (also known as sensitivity or true positive rate) indicates the proportion of observed charged off loans correctly predicted by the model:

$$
\text { Recall }=\frac{\text { Charged off predicted as such }}{\text { All charged off loans }}
$$


Precision complements recall by showing the proportion of correctly predicted charged off loans with respect to the total predicted charged off:

$$
\text { Precision }=\frac{\text { Charged off predicted as such }}{\text { All predicted charged off loans }}
$$

Recall is not so much about capturing cases correctly, but more about capturing all cases which fall into the category of interest to us. Predicting all loans as being charged off would give a $100 \%$ recall. On the other hand, Precision focuses on minimizing false positives, which in an ideal case would lead to having a $100 \%$ precision. Generally, the aim is to balance Precision and Recall in such a way that the false predictions are minimal and the true ones are maximized. To this aim, we will want both Recall and Precision to be as high as possible.

It is imperious to lower the probability of allowing non-performant loans, while keeping the number of applications mistakenly rejected to a minimum. Otherwise, the lenders will be losing money and the platform clients, as a high rejection rate would eventually have a negative impact on the business.

Having to consider both goals, a useful measure for seeking a balance between precision and recall, particularly in the context of unbalanced data, is the F1-score:

$$
F 1-\text { score }=2 \cdot \frac{\text { Precision } \cdot \text { Recall }}{\text { Precision }+ \text { Recall }}
$$

This measure is the harmonic mean of Recall and Precision and it is a very useful indicator of model performance since it takes into account both the interests of the lenders (good identification of bad loans) and those of the platform (reducing the chances to classify an application as bad when it is not).

Another way to assess model performance is to visualize all (Recall, FPR) pairs at various thresholds. The resulting figure is called ROC (Receiver-Operator Characteristic) curve, where FPR (False Positive Rate) is:

$$
F P R=\frac{\text { Fully paid loans predicted as charged off }}{\text { All fully paid loans }}
$$

After plotting the data, we are interested in the area under the ROC curve, which is known as AUC (Area Under the Curve). This value indicates the model's ability to distinguish between good and bad clients. A model that perfectly predicts the two categories will have an AUC of 1. Conversely, if the model makes no distinction between the two classes (it assigns them randomly to good and bad), the value of AUC will be 0.5. It is unlikely that the AUC would go below 0.5, but in case it does, this suggests a problem in coding the data, since the model classifies cases in the opposite category, namely the good ones as bad and the other way around. 


\section{Results and discussions}

As the number of variables considered in the sample is relatively large, we will focus on the most correlated variables with the target, since they are most likely to have an impact in the final model. To this aim, Table 1 presents the strongest five correlations, regardless of their direction, for each of the five treatment approaches considered, ordered in ascending order after the values for no outlier treatment. All correlations are highly statistically significant, with p-values below 0.001 .

The first four variables are the same for all approaches, but for binning and replacement

with median loan grade has a stronger correlation than interest rate. The fifth variable is DTI for all approaches except no outlier treatment, for which it is the number of mortgage accounts instead. The consistency between approaches regarding the predictors that are most correlated with the target suggests the importance of the respective variables in predicting the loan status.

Table 1. Top five variable based on Pearson correlation coefficient for each approach

\begin{tabular}{|l|c|c|c|c|c|c|c|c|c|c|}
\hline \multirow{2}{*}{\multicolumn{1}{c|}{ variable }} & \multicolumn{2}{c|}{ No treatment } & \multicolumn{2}{c|}{ Deleted } & \multicolumn{2}{c|}{ Binned } & \multicolumn{2}{c|}{ Median } & \multicolumn{2}{c|}{ Winsorized } \\
\cline { 2 - 12 } & correl & p-val & correl & p-val & correl & p-val & correl & p-val & correl & p-val \\
\hline fico_score & -0.756 & $<0.001$ & -0.756 & $<0.001$ & -0.654 & $<0.001$ & -0.756 & $<0.001$ & -0.757 & $<0.001$ \\
\hline grade & 0.276 & $<0.001$ & 0.262 & $<0.001$ & 0.267 & $<0.001$ & 0.254 & $<0.001$ & 0.277 & $<0.001$ \\
\hline int_rate & 0.274 & $<0.001$ & 0.258 & $<0.001$ & 0.274 & $<0.001$ & 0.274 & $<0.001$ & 0.274 & $<0.001$ \\
\hline term_60 months & 0.151 & $<0.001$ & 0.141 & $<0.001$ & 0.151 & $<0.001$ & 0.151 & $<0.001$ & 0.151 & $<0.001$ \\
\hline mort_acc & -0.096 & $<0.001$ & & & & & & & & \\
\hline dti & & & 0.109 & $<0.001$ & 0.111 & $<0.001$ & 0.113 & $<0.001$ & 0.115 & $<0.001$ \\
\hline
\end{tabular}

We see that the correlation coefficients differ slightly from one approach to another. Winsorization has highest coefficients in all cases and elimination of outliers generally tends to have the lowest one.

The strongest correlation in all cases is for the FICO score, which is also the only negative one in the top five that is common to all approaches. This suggests that we can expect lower scores to be associated with higher probabilities of default. Indeed, according to the description provided by the developers of the model, FICO ranges between 300 and 850 and the lower the score, the riskier the applicant is. This score is widely used in banking and P2P lending because it is based on various information collected by credit bureaus, such as financial habits, timely utility payments or store transactions for several years. Figure 1 confirms that lower FICO scores indicate a higher risk applicant. What is more, the difference between medians is around 150 points and only a quarter of the fully paid loans have similar FICO scores as $75 \%$ of the charges of ones.

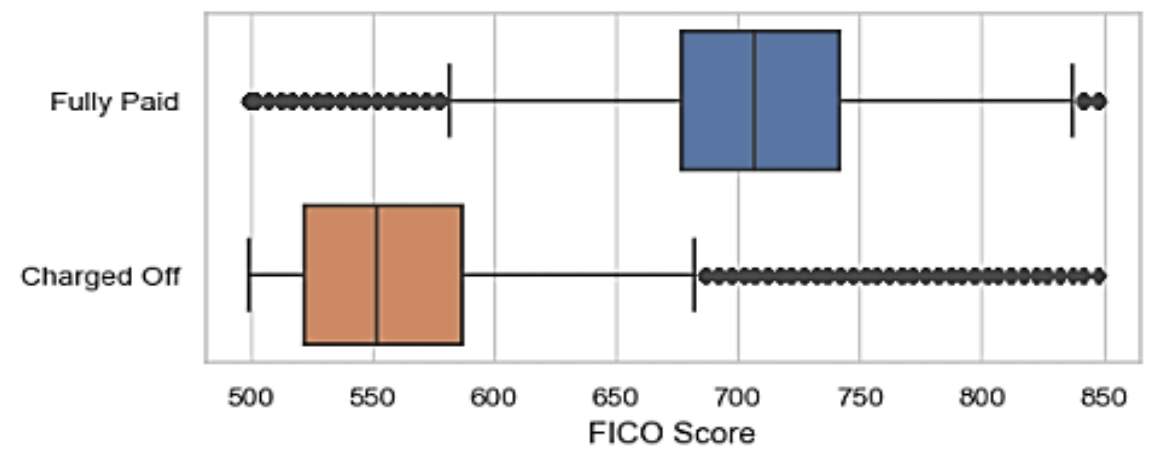

Figure 1. FICO score by loan status

Source: Authors' own research. 
The loan grading system allows lending institutions to classify clients in groups with similar characteristics. Grading is part of an institution's risk mitigation strategy and it is created to cluster clients based on information like credit history, repayment likelihood or collateral quality. In Lending Club's case there are seven grades, labeled from A to G, where A represents lowest risk. During data preprocessing, we recorded this variable such that as the risk increases, so does the variable code. Thus, $A$ is coded with 1 and $G$ with 7 . Figure 2 confirms the relationship between the grade and the final loan status, with less than $10 \%$ of the borrowers with grade A having been charged off as opposed to about $55 \%$ of those with grade $\mathrm{G}$.

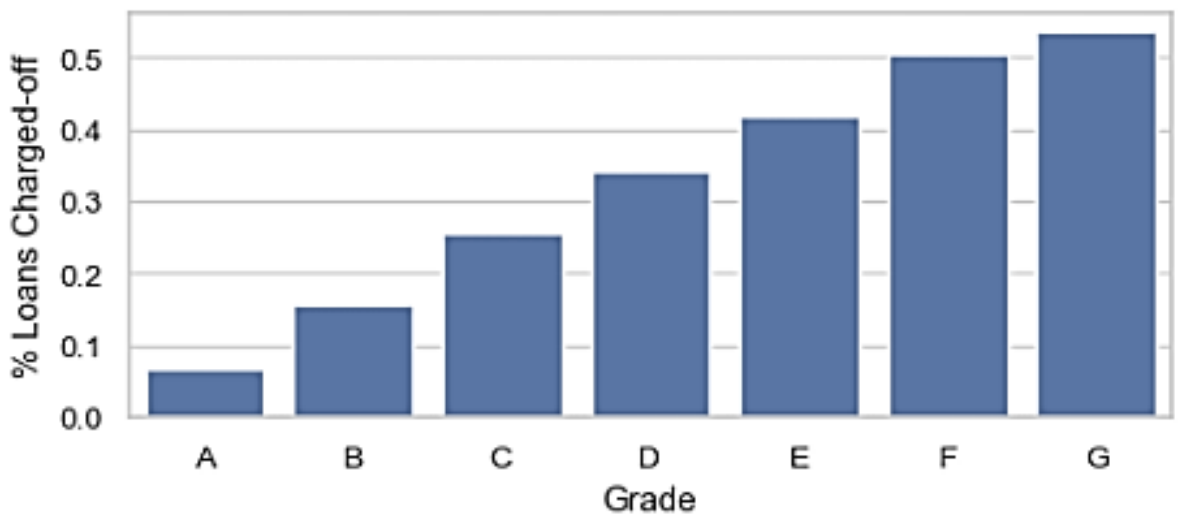

Figure 2. Charge off rate by grade

Source: Authors' own research.

The relationship between interest rate and probability of default is predictable: loans with higher interest rates are more likely to default than those with lower interest rates. Figure 3 confirms this relationship and also shows that variability in interest rates is high within both groups, but to a greater extent in the charged-off category. Half of the fully repaid loans have interest rates of about $11.5 \%$ or less while in the case of charged off loans, $75 \%$ of them paid at least $12 \%$ interest. Similarly, the median value for the charged off group is close to the third quartile for the fully paid group.

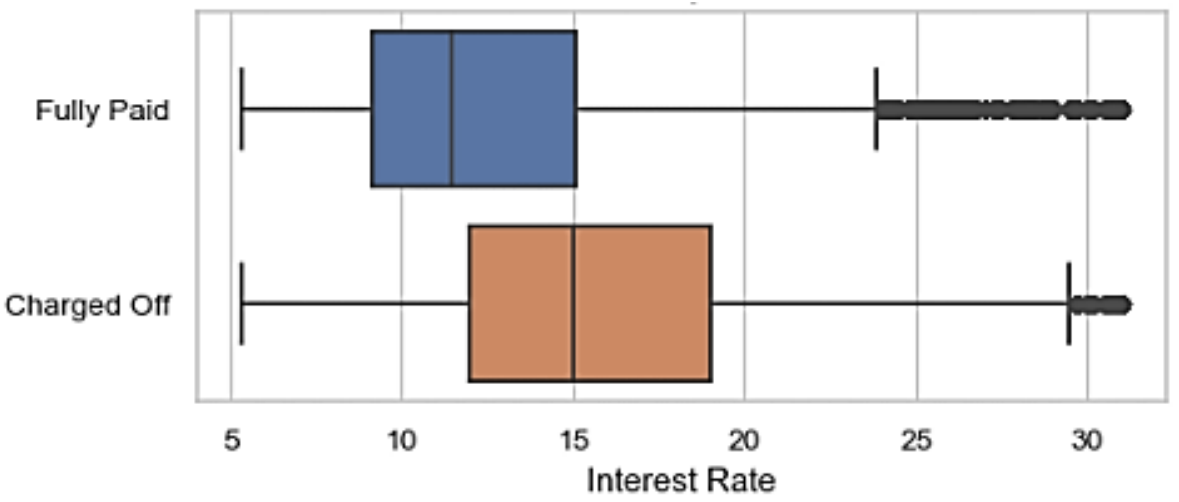

Figure 3. Interest rate by loan status

Source: Authors' own research.

The term variable is binary as the loans were given for either 36 or 60 months. The longer the credit period, the higher the risk of default, because on a longer term, among others, 
macroeconomic fluctuations can have a stronger impact. In our data, about 4 out of 5 loans are for 36 months, and the default rate is almost double for loans on longer term (Figure 4).

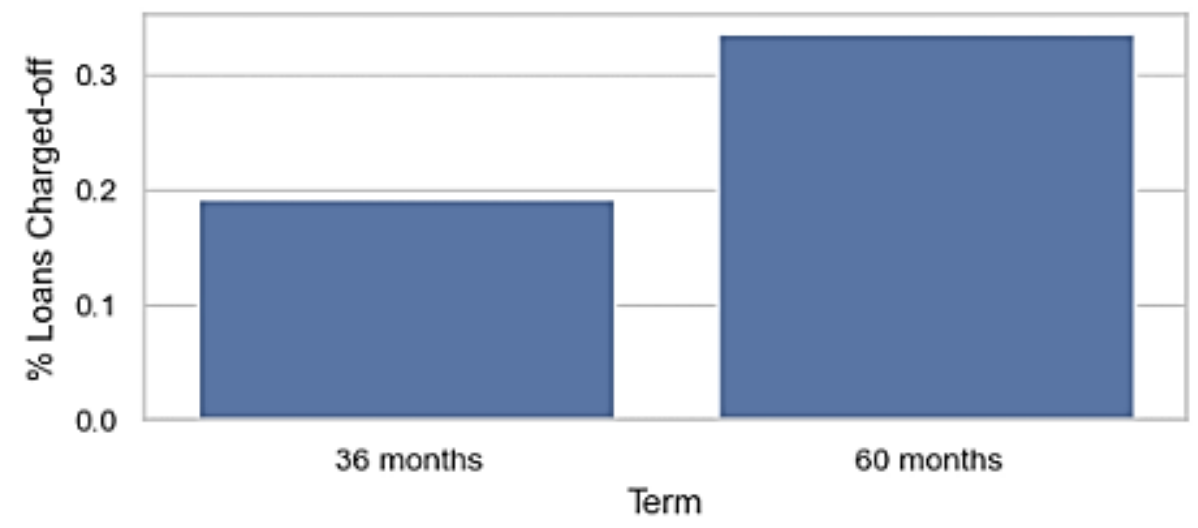

Figure 4. Charge off rate by term

Source: Authors' own research.

The results for debt-to-income ratio (DTI) differ to a lesser degree between the groups, which is also reflected in the low correlation coefficient. The difference between the medians of the two groups is about $2.35 \%$ and in variation of $0.86 \%$.

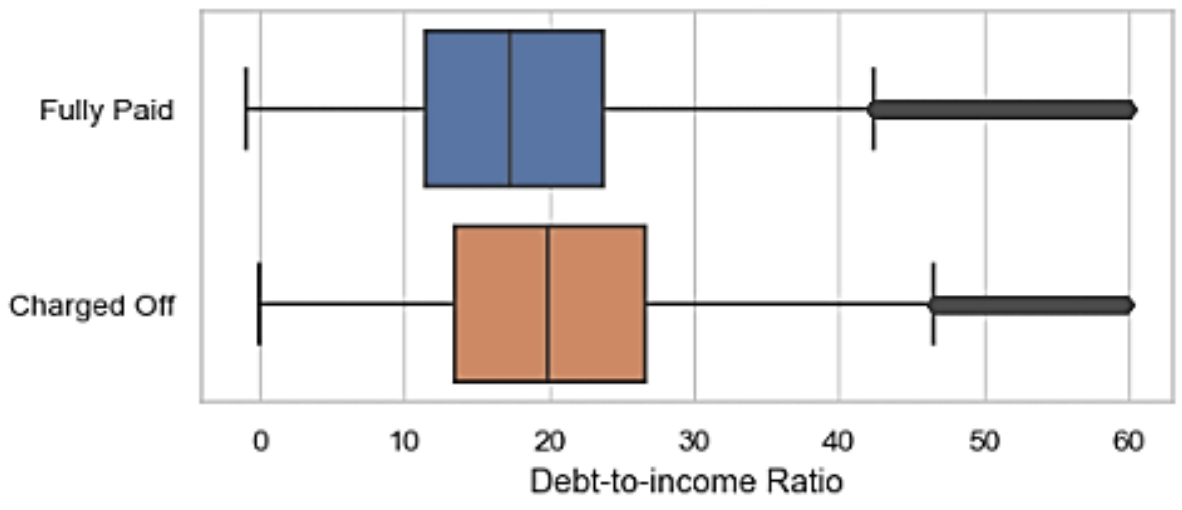

Figure 5. Debt-to-income ratio by loan status

Source: Authors' own research.

Number of mortgage accounts tends to be a bit higher for fully paid loans (1.65 on average) than for charged off ones (1.22 on average), but standard deviation is higher than the mean for both groups so the difference might be non-significant. Other variables that might prove important in estimating the probability of default are the borrowed amount, the annual income of the borrower (log-transformed) and whether the applicant is a homeowner.

Aside from the correlations between target and predictors, we also assessed the correlations between predictors in order to detect possible multicollinearity issues. Based on the results, the only correlation was between loan amount and installment. Regardless of the approach, the two are essentially equally correlated to the loan status, so we kept the loan amount because it was cleaner. 


\section{Outlier treatment approaches}

Starting from the outlier definition, we identified them for each continuous variable (Table 2). The charged off rate in the training sample is $23.3 \%$ with outliers present (regardless of treatment applied) and $22.3 \%$ after eliminating all outliers, which suggests that outliers play an important role in identifying charged off loans.

In comparison to the entire portfolio, the number of outliers is low, the highest percentage being present in the variable indicating the revolving balance (log-transformed), for which the correlation coefficient with the target variable is basically 0 regardless of the approach, so treatment of the outliers in this variable will most likely have no impact on the model. Among the three continuous variables we identified as strongest predictors, interest rate has a relatively large number of outliers $(2.66 \%)$. In this case, no treatment and winsorizing lead to the highest correlation coefficients (0.28) and replacement with median to the lowest (0.25), but the impact is small overall. For DTI, the coefficient varies between 0.09 and 0.015 , but the impact could be quite big, considering the very small number of outliers in this variable. FICO score has no outliers so the only change noticed is between binned and non-binned approaches.

Table 2. Percent of outliers in the continuous variables

\begin{tabular}{|l|r|}
\hline \multicolumn{1}{|c|}{ Variable } & Outliers \\
\hline Revolving balance (log) & $3.83 \%$ \\
\hline Number of open accounts & $2.73 \%$ \\
\hline Interest rate & $2.66 \%$ \\
\hline Number of accounts opened in the past 24 months & $2.24 \%$ \\
\hline Annual income (log) & $1.89 \%$ \\
\hline Total number of accounts & $1.78 \%$ \\
\hline Number of mortgage accounts & $0.97 \%$ \\
\hline Loan amount & $0.82 \%$ \\
\hline DTI & $0.58 \%$ \\
\hline Revolving utilized & $0.01 \%$ \\
\hline FICO score & $0.00 \%$ \\
\hline Employment length & $0.00 \%$ \\
\hline
\end{tabular}

Source: Authors' own research.

Further we present an analysis for the impact each of the three methods of dealing with outliers, namely binning, replacement with median and winsorizing, has on the input data.

To ensure no data leakage, binning cut-off values were obtained from the training sample and applied on the test sample. For this reason, most variables have roughly $20 \%$ of the loans in each bin for the training sample. The data in the test sample is less uniformly distributed, but generally the deviations are less than $3 \%$ in either direction.

In case of many variables, the first bin is bigger in the test sample which indicates a tendency towards concentration of values. This is particularly obvious for variables regarding revolving balance and utilization. The amount utilized decreased, leading to a bin that is almost $12 \%$ bigger in the test sample than in the training data and most of the shift affected the last two bins, namely the ones with high amounts. Similarly, the revolving balance (log) is decreasing leading to about $7.5 \%$ more clients in the first bin, with increasing contributions from superior bins. On the other end of the spectrum, the number of clients with higher FICO scores increased, indicating that the creditworthiness of potential borrowers increased over time. 
Table 3. Percentage of observations in each bin for continuous variables

\begin{tabular}{|l|c|c|c|c|c|c|c|c|c|c|}
\hline & \multicolumn{4}{|c|}{ Training sample } & \multicolumn{4}{c|}{ Test sample } \\
\cline { 2 - 12 } & $\mathbf{0}$ & $\mathbf{1}$ & $\mathbf{2}$ & $\mathbf{3}$ & $\mathbf{4}$ & $\mathbf{0}$ & $\mathbf{1}$ & $\mathbf{2}$ & $\mathbf{3}$ & $\mathbf{4}$ \\
\hline Loan amount & 20.16 & 22.43 & 19.95 & 17.54 & 19.91 & 23.28 & 22.16 & 17.86 & 15.68 & 21.02 \\
\hline Interest rate & 20.33 & 20.81 & 20.54 & 18.82 & 19.49 & 19.35 & 20.68 & 17.37 & 18.20 & 24.39 \\
\hline Employment length & 25.50 & 15.14 & 23.25 & 36.12 & & 27.07 & 15.46 & 21.90 & 35.56 & \\
\hline DTI & 20.03 & 19.98 & 20.03 & 19.98 & 19.98 & 24.52 & 19.56 & 18.74 & 17.75 & 19.42 \\
\hline Number of open accounts & 22.38 & 25.18 & 14.83 & 19.87 & 17.74 & 26.36 & 24.75 & 14.03 & 18.47 & 16.40 \\
\hline Revolving utilized & 20.02 & 20.02 & 20.06 & 19.94 & 19.96 & 31.81 & 20.41 & 17.19 & 15.45 & 15.14 \\
\hline Total number of accounts & 20.01 & 21.08 & 20.45 & 19.45 & 19.02 & 23.69 & 21.38 & 19.47 & 17.94 & 17.52 \\
\hline Accounts opened in the past 24 months & 23.17 & 27.62 & 12.19 & 17.65 & 19.36 & 23.49 & 27.08 & 12.03 & 17.58 & 19.82 \\
\hline Number of mortgage accounts & 74.30 & 10.93 & 14.78 & & & 75.92 & 10.68 & 13.40 & & \\
\hline Annual income (log) & 20.26 & 23.03 & 17.06 & 19.65 & 20.00 & 21.88 & 22.57 & 16.60 & 19.07 & 19.89 \\
\hline FICO score & 20.32 & 20.87 & 21.78 & 17.30 & 19.73 & 15.31 & 17.98 & 24.18 & 19.75 & 22.78 \\
\hline Revolving balance (log) & 20.01 & 20.00 & 20.00 & 20.00 & 20.00 & 27.39 & 19.19 & 18.17 & 17.88 & 17.36 \\
\hline
\end{tabular}

PICBE |

For two of the variables some of the cut-off points were equal, so the resulting bins were merged. In case of employment length about a third of the loans were granted to applicants with 10 or more years of experience. The variable did not record the actual number of years, all experience above 9 years being placed in a "10+ years" category. For this reason, the last two bins were merged. Similarly, a high number of applicants have no mortgage accounts, so the first three bins are merged into a single one, which is why the concentration is high in the first bin.

Dealing with outliers by replacing them with the median leads to smaller means and standard deviations (Table 4), particularly for installment, number of accounts (all three types) and number of mortgage accounts. In the case of DTI, the mean decreases with about $1.5 \%$ while the standard deviation drops to about $74 \%$ of the initial value. At the opposite pole is revolving amount utilized, where there is no impact in the mean and negligible impact in the standard deviation. This is most likely explained by the very small number of outliers in the variable $(0.01 \%)$.

Table 4. Changes in mean and standard deviation after replacing outliers with median

\begin{tabular}{|l|r|r|r|r|}
\hline \multirow{2}{*}{} & \multicolumn{2}{|c|}{ Mean } & \multicolumn{2}{c|}{ St Dev } \\
\cline { 2 - 5 } & \multicolumn{1}{|c|}{ initial } & \multicolumn{1}{c|}{ new } & initial & \multicolumn{1}{c|}{ new } \\
\hline Loan amount & 144367 & 14207 & 9005 & 8702 \\
\hline Interest rate & 13.28 & 12.87 & 5.16 & 4.52 \\
\hline DTI & 18.73 & 18.45 & 11.37 & 8.43 \\
\hline Number of open accounts & 11.88 & 11.35 & 5.74 & 4.75 \\
\hline Revolving utilized & 48.99 & 48.99 & 24.34 & 24.33 \\
\hline Total number of accounts & 24.83 & 24.08 & 12.15 & 10.76 \\
\hline Accounts opened in the past 24 months & 5.03 & 4.75 & 3.38 & 2.85 \\
\hline Number of mortgage accounts & 1.57 & 1.49 & \multicolumn{3}{|c|}{1.87} & 1.69 \\
\hline
\end{tabular}

In contrast, winsorizing has little to no impact on the means (Table 5), but it still has significant impact in DTI standard deviation and some effect on variation in the number of various types of accounts (all, mortgage, open, opened in the past 24 months). This suggests that in DTI, despite being few, the outliers deviate very much from the rest of the data. 
Table 5. Changes in mean and standard deviation after winsorizing outliers

\begin{tabular}{|c|c|c|c|c|}
\hline & \multicolumn{2}{|c|}{ Mean } & \multicolumn{2}{|c|}{ St Dev } \\
\hline & initial & new & initial & new \\
\hline Loan amount & 14437 & 14406 & 9005 & 8910 \\
\hline Interest rate & 13.28 & 13.26 & 5.16 & 5.12 \\
\hline Installment & 445.99 & 445.44 & 278.71 & 276.46 \\
\hline DTI & 18.73 & 18.57 & 11.37 & 8.55 \\
\hline Number of open accounts & 11.88 & 11.83 & 5.74 & 5.51 \\
\hline Revolving utilized & 48.99 & 48.98 & 24.34 & 24.27 \\
\hline Total number of accounts & 24.83 & 24.75 & 12.15 & 11.78 \\
\hline Accounts opened in the past 24 months & 5.03 & 5 & 3.38 & 3.24 \\
\hline Number of mortgage accounts & 1.57 & 1.55 & 1.87 & 1.78 \\
\hline FICO score & 671.77 & 671.69 & 81.99 & 81.84 \\
\hline
\end{tabular}

PICBE |

One thing to note is that we excluded from tables 4 and 5 information for some variables (earliest credit line date, employment length, the two log-transformed variables and FICO score). Since the earliest credit line date is a time variable, it does not make sense to change its values; outliers are simply clients with a very long credit history. We removed duration of employment and the FICO score from the list of variables to be treated with replacement by median or winsorization because the variables have no outliers (see Table 2).

\section{Modelling results}

Based on the results in Figure 6, all models performed well, with test balanced accuracies ranging between 0.85 and 0.90 and F1 scores revolving around 0.85 . For balanced accuracy and precision, the approach regarding the outliers had little impact on the metric stability, the min-max interval from training being small. On the other hand, test scores vary closer to a 0.1 range for F1-scores and Recall.

Except for precision and ROC-AUC, the test metrics are generally within the interval observed during testing, which is expected behavior. ROC-AUC test results are lower than during training, which is not uncommon and it might suggest the model is underfitting the data. Conversely, precision test scores are much higher than during training. A possible explanation for this is that in the case of class imbalance, precision is not a good metric to assess model performance since the model will probably predict few cases from the smaller class, many of which will be from the respective class. In this case precision will be high, despite the model not being very good at identifying the small class.

An easily noticeable conclusion is that the binning approach performs poorer than the others in all training metrics, which suggests this might not be the best option when dealing with outliers. Information loss caused by the binning process might be the culprit, particularly in the context of imbalanced classes and having may outliers in the smaller class, which is also the one of interest. What this finding tell us is that when outliers are present, binning should be considered as an option, but employed in the final model only as last resort.

This conclusion challenges the generally used approach in banking, for example, where binning is used as a standard rather than being assessed against other options. While it can prove extremely useful in certain cases, like extreme class imbalance and small datasets, winsorization seems to be a viable alternative that would neither require dropping precious information, nor would it alter the relationships in the data. 


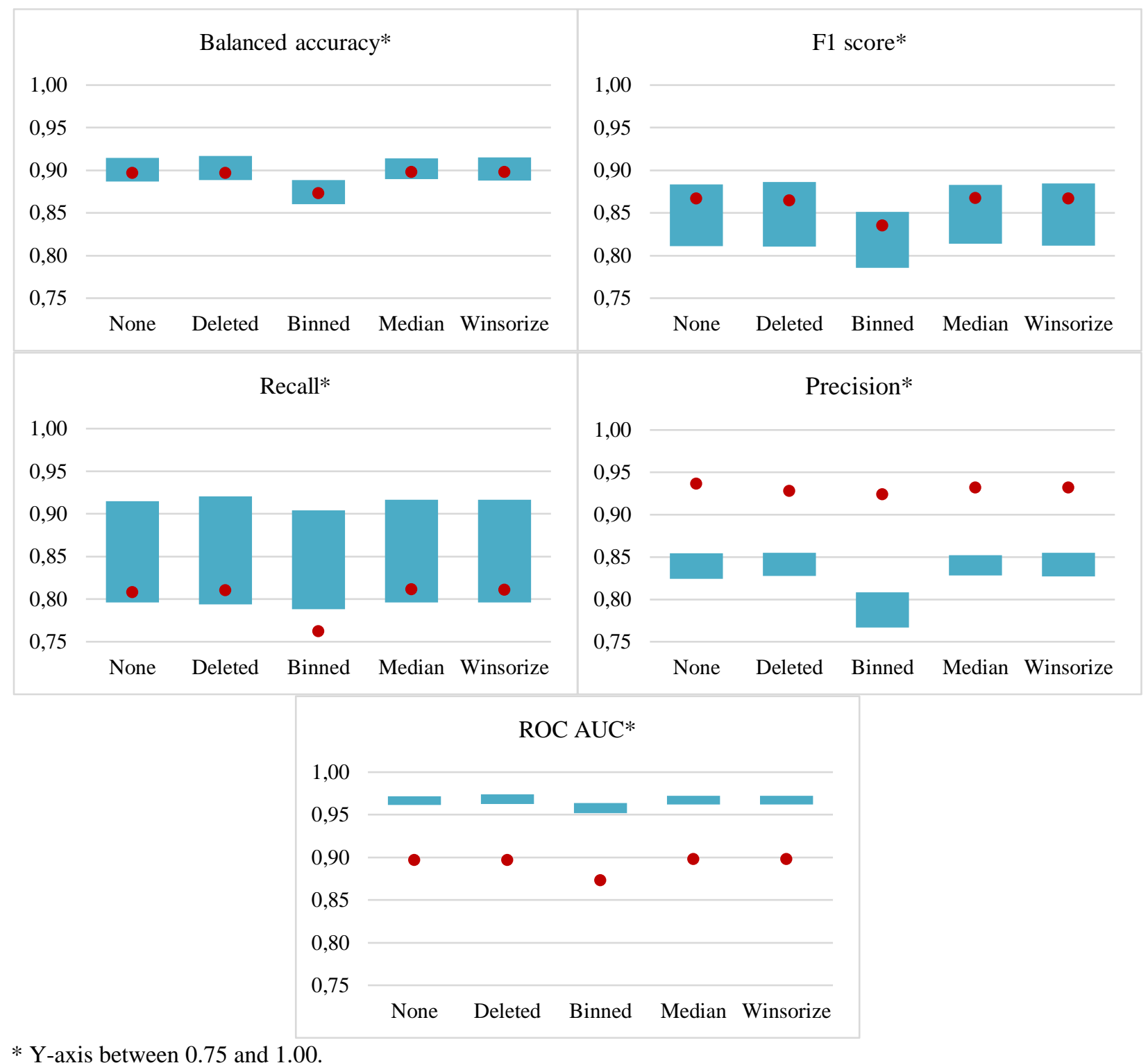

PICBE |

* Y-axis between 0.75 and 1.00 .

Figure 6. Test scores (red dots) and training results min-max interval (blue stripes) for 10-fold CV

Source: Authors' own research.

We analyzed the strongest predictors for each model to see to what extent the results are consistent. For all models, the higher the amount borrowed and the DTI, as well as taking a loan for a 60-month period had the biggest negative impact, while a higher FICO score, annual income and utilized amount, together with a longer period since opening a credit line, were the main risk reducing factors. Other predictors that appear frequently and which also increase the risk of default are the interest rate and taking a loan for debt consolidation. 

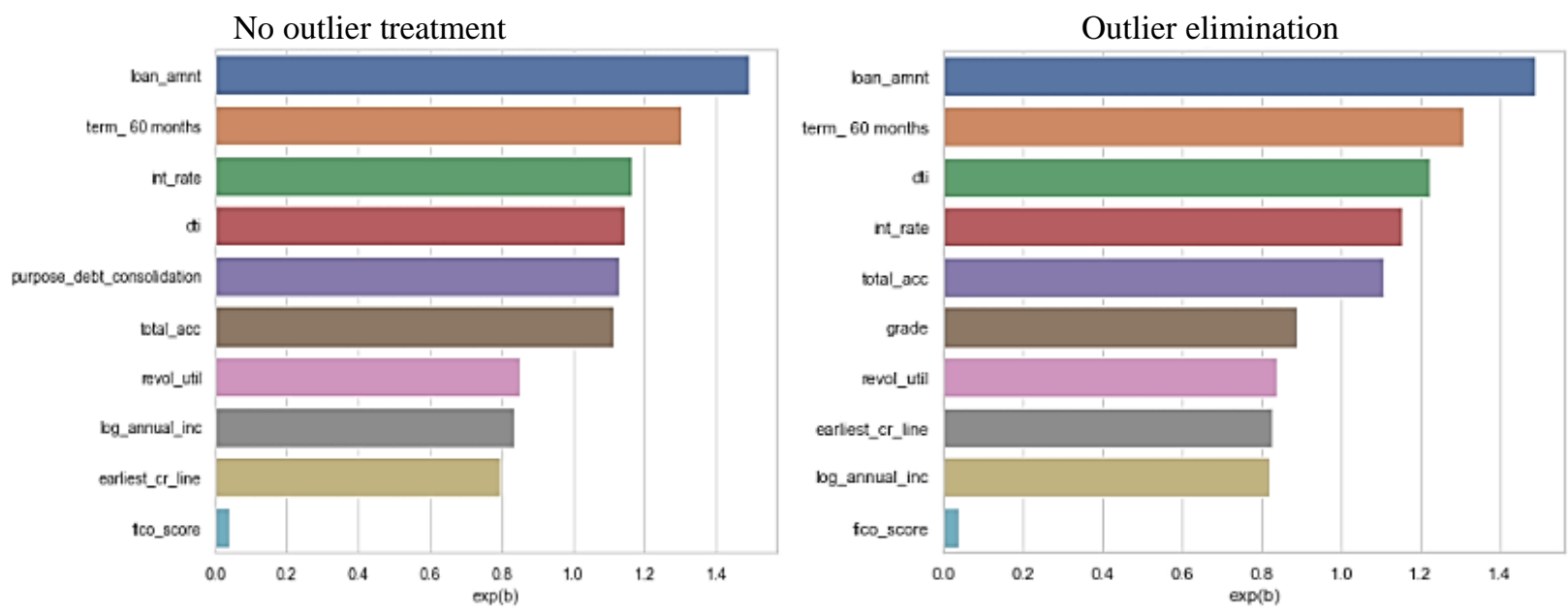

PICBE |
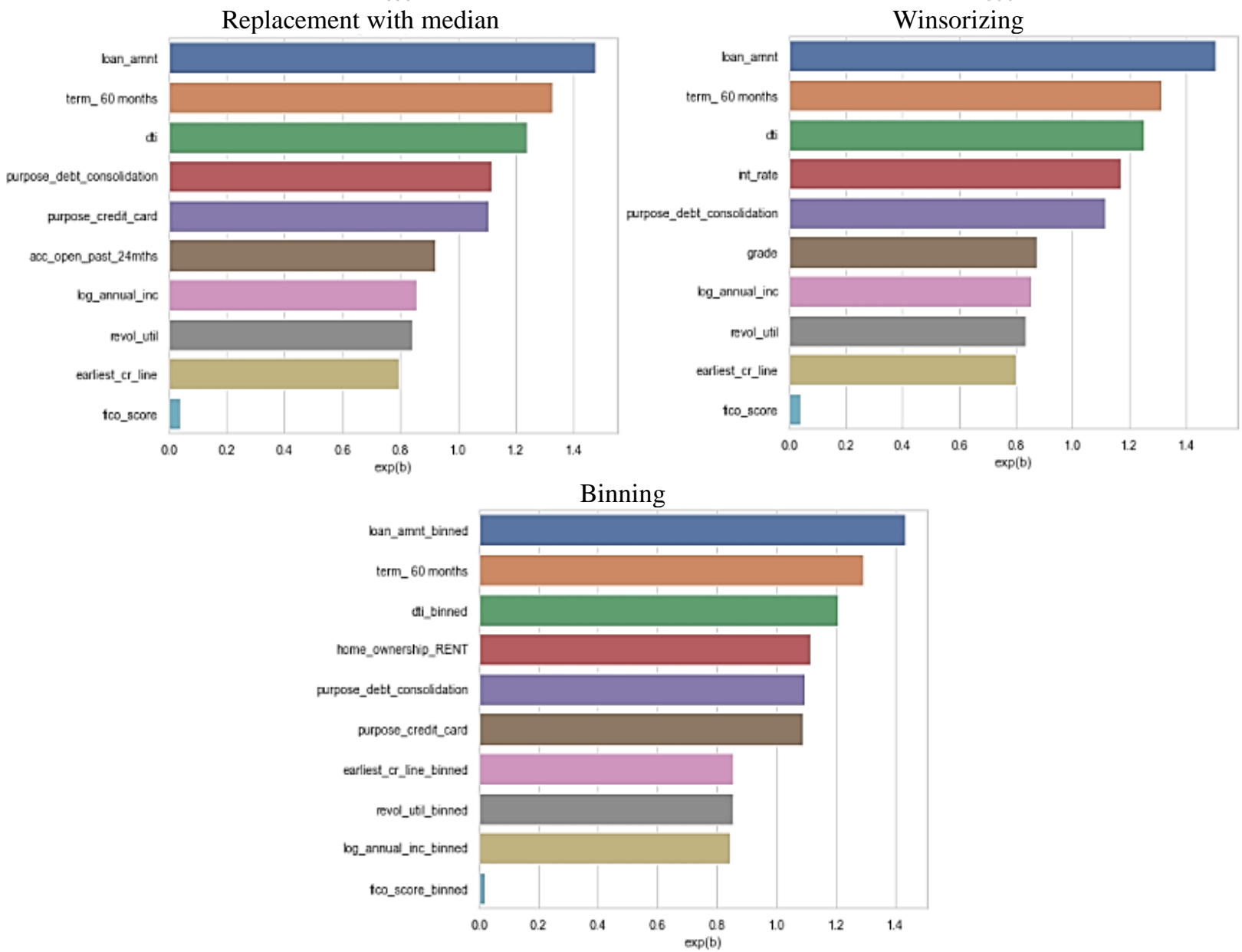

Figure 7. Strongest ten predictors in the logistic regression model

Source: Authors' own research.

The list of predictors is both fairly consistent and it makes sense from a business standpoint so we can conclude that the chosen approach will likely not significantly impact the model from a business perspective. Yet, there is variation in the list of top ten predictors for the five models and 
the binning approach allows for more categorical predictors in the model compared to the other ones. It follows that a thorough analysis is necessary before deciding on how to proceed when outliers are present in the data and the opportunity to apply several methods, depending on the variable, as opposed to batch-treatment should be considered.

\section{Limitations and future research}

Some of the approaches proposed can alter the relationship of the predictors with the target variable. As we saw in the brief analysis describing the outlier treatment approaches from the results section, applying them on data had an impact both on variables with outliers and in the ones with no outliers (FICO).

Future studies might focus on outlier impact in the context of class imbalance. For large datasets as the Lending Club one, under-sampling, while not desirable, is feasible, but in case of smaller portfolios this is a luxury most cannot afford (Nonso Nnamoko, Ioannis Korkontzelos). On the other hand, over-sampling comes with the risk of increasing the outliers in the sample, which also impacts model performance. Performed properly, though, it can improve the model's ability to correctly classify an application by making outliers less infrequent (Chawla). We believe this problem must be analyzed to propose approaches for even better preprocessing for good modelling data quality.

As opposed to univariate outliers, which are extreme and unusual values in one variable, multivariate outliers are combinations of unusual scores on several variables. This type of outliers may result from the combination of 'normal' values on the variables involved, so dealing with univariate outliers does not necessarily imply all outliers have been assessed and treated. For example, in the relationship between DTI, interest rate and loan status, we might expect charged off loans to have high interest rates and high DTIs, so having a charged off client with particularly small interest rate and DTI would be unusual in the multivariate context, despite the low values being "normal" in the univariate analysis. Further research in this direction could lead to more robust estimations using consecrated methods, as opposed to focusing on developing more complex modelling methods.

Conversely, while data quality should remain an important criterion, identification of alternative modelling techniques that are robust to outliers should be pursued. Transparency and ease of use allows both regulators and lenders to rely on logistic regression, but other powerful methods are being proposed. An example would be the decision-tree based ones, like random forests with boosting and bagging (Coser, Maer-Matei, \& Albu, 2019; Nabende \& Senfuma, 2019; Ramakrishnan, Mirzaei, \& Bekri, 2015). These models are also transparent and accessible, but, unlike the logistic regression, they make no assumptions regarding the distribution of the data and are robust to outliers. On the other hand, several studies have found that logistic regression outperforms models based on this method, particularly when there is class imbalance, as is always the case for probability of default models (Abdemoula, 2015; Coser, Maer-Matei, \& Albu, 2019; Çı̆̆şar \& Ünal, 2019).

Other directions explored are Support Vector Machines (SVM) (Härdle, Moro, \& Schäfer, 2007; Obare \& Muraya, 2018) and neural networks (NN) (Bayraci \& Susuz, 2019; Turiel \& Aste, 2020), but their support from regulators is currently marginal due to their lack of transparency. SVM relies on identifying the cases generating a separation line between classes, which does not allow lenders to create profiles for borrowers. NN are even less transparent in the sense that they are black-box models so further research in making them more explainable might lead to increased acceptance in the lending sector. 


\section{Conclusion}

In the context of P2P lending platforms, the power dynamic between stakeholders is different from traditional banking. The entire process is intermediated by a platform, the owners of which have as their main priority ensuring they have sufficient investors to finance the loans on one hand and having as many financed applications as possible on the other hand. While not directly affected by borrower creditworthiness, since the risk is entirely borne by the lender, the platform still needs to check applicants, lest risk withdrawal of investors which would lead to business closure. For this reason, platforms adopted the grading system in traditional banking and are providing potential investors with a risk profile of the applicant. This way each investor can decide which loan to fund and to what extent based on their own risk appetite.

Probability of default models can be used by investors to assess the risk entailed by a potential investment and they can play a major role in the decision regarding financing. To this end, the quality of the model has a great impact on the investor's outcome and a performant model is highly dependent on the quality of its input data. While most models are based on data that is analyzed from univariate and multivariate perspectives, an often overlooked aspect is the analysis of outliers, followed by their treatment according to the modelling objectives. Depending on the results, one or more approaches may be considered and tested, starting with concluding that their impact is not significant in the available sample (no treatment), considering eliminating them from the sample altogether, binning or replacing them with other values. Alternatively, models that are robust to outliers can be chosen instead of the industry standard.

We explored several methods for dealing with outlier presence and described their advantages and disadvantages in the context of probability of default estimation for credit risk. The results obtained lead us to challenge the common approach used in traditional banking, namely binning, and advocate for alternative approaches that are viable alternatives and do not involve losing information or altering the data. The best approach depends on many factors, from sample size and class (im)balance, to variable distribution in the univariate space and combinations of variables for multivariate analyses. More importantly, several methods should be considered and tested for each variable in order to find the optimal strategy for preparing the data for modelling.

\section{Acknowledgements}

This research has received funding from the European Union's Horizon 2020 research and innovation program FIN-TECH: A Financial supervision and Technology compliance training programme under the grant agreement No. 825215 (Topic: ICT35-2018, Type of action: CSA). The research has also received funding from the COST European Cooperation in Science and Technology under the action CA19130 - Fintech and Artificial Intelligence in Finance - Towards a transparent financial industry.

\section{References}

Abdemoula, A. K. (2015). Bank Credit Risk Analysis with K-Nearest-Neighbor Classifier: Case of Tunisian Banks. Accounting and Management Information Systems, 14(1), 79-106.

Akcura, K., \& Chhibber, A. (2018). Design and Comparison of Data Mining Techniques for Predicting Probability of Default on a Loan. Retrieved from https://www.researchgate.net/ profile/Korhan_Akcura2/publication/335173357_Design_and_Comparison_of_Data_Min ing_Techniques_for_Predicting_Probability_of_Default_on_a_Loan/links/5d54a79d9285 1c93b630b8be/Design-and-Comparison-of-Data-Mining-Techniques-for-Pr. 
Bayraci, S., \& Susuz, O. (2019). A Deep Neural Network (DNN) based Classification Model in Application to Loan Default Prediction. Theoretical and Applied Economics, XXV(4(621)), 75-84.

Chawla, N., Bowyer, K., Hall, L., \& Kegelmeyer, W. (2002). Smote: synthetic minority oversampling technique. Journal Of Artificial Intelligence Research, 16, 321-357. doi:DOI: 10.1613/jair.953.

Çığşar, B., \& Ünal, D. (2019). Comparison of Data Mining Classification Algorithms Determining the Default Risk. Scientific Programming, 1-8.

PICBE |

Coser, A., Maer-Matei, M., \& Albu, C. (2019). Predictive Models for Loan Default Risk Assessment. Economic Computation and Economic Cybernetics Studies and Research, 53, 149-165. doi:10.24818/18423264/53.2.19.09.

Green, R. F. (1976). Outlier-Prone and Outlier-Resistant Distributions. Journal of the American Statistical Association, 71(354), 502-505. doi:10.2307/2285341.

Gurný, P., \& Gurný, M. (2013). Comparison of Credit Scoring Models on Probability of Default Estimation for Us Banks. Prague Economic Papers, 22(2), 163-181. doi:10.18267/ j.pep.446.

Härdle, W. K., Moro, R. A., \& Schäfer, D. (2007). Estimating probabilities of default with support vector machines. Discussion Paper Series 2: Banking and Financial Studies, Deutsche Bundesbank.

Hadad, S., and Bratianu, C. (2019). "Dematerialization of banking products and services in the digital era". Management \& Marketing. Challenges for the Knowledge Society, Vol. 14, Issue 3, 318-337.

Hawkins, D. M. (1980). Identification of outliers (Monographs on applied probability and statistics). Chapman\&Hall. doi:https://doi.org/10.1007/978-94-015-3994-4.

Kaya, M. E., Gurgen, F., \& Okay, N. (2008). An Analysis of Support Vector Machines for Credit Risk Modeling. Proceedings of the 2008 conference on Applications of Data Mining in EBusiness and Finance, 25-33. IOS Press.

Lending Club (n.d.). database. Retrieved March 2, 2021, from https://www.kaggle.com/ wordsforthewise/lending-club.

Nabende, P., \& Senfuma, S. (2019). A study of machine learning models for predicting loan status from Ugandan loan applications. Athens.

Nh, N., \& Mai, N. (2018). Naïve Bayesian Classifier and Classification Trees for the Predictive Accuracy of Probability of Default Credit Card Clients. American Journal of Data Mining and Knowledge Discovery, 3(1), 1-12. Retrieved from http://article.sciencepublishing group.com/pdf/10.11648.j.ajdmkd.20180301.11.pdf.

Nnamoko, N., \& Korkontzelos, I. (2020). Efficient treatment of outliers and class imbalance for diabetes prediction. Artificial Intelligence in Medicine, 104. doi:https://doi.org/10.1016/ j.artmed.2020.101815.

Obare, D., \& Muraya, M. (2018). Comparison of Accuracy of Support Vector Machine Model and Logistic Regression Model in Predicting Individual Loan Defaults. American Journal of Applied Mathematics and Statistics, 6(6), 266-271. doi:10.12691/ajams-6-6-8.

Pop, I. D., Chicu, N., Radutu, A. (2018). "Non-performing loans decision making in the Romanian banking system". Management \& Marketing. Challenges for the Knowledge Society, Vol. 13, Issue 1, 761-776. 
Ramakrishnan, S., Mirzaei, M., \& Bekri, M. (2015). Corporate Default Prediction with AdaBoost and Bagging Classifiers. Jurnal Teknologi, 73(2), 45-50. Retrieved from https://jurnal teknologi.utm.my/index.php/jurnalteknologi/article/view/4191/2954.

Sariev, E., \& Germano, G. (2020). Bayesian Regularized Artificial Neural Networks for the Estimation of the Probability of Default. Quantitative Finance, 20(0), 311-328.

Sharma, P. K., Haleem, H., \& Ahmad, T. (2015). Improving Classification by Outlier Detection and Removal. In S. C. Satapathy, A. Govardhan, K. S. Raju, \& J. K. Mandal (Ed.), Emerging ICT for Bridging the Future - Proceedings of the $49^{\text {th }}$ Annual Convention of the Computer Society of India CSI Volume 2, 621-628. Cham: Springer International Publishing. doi:10.1007/978-3-319-13731-5_67.

Smith, M. R., \& Martinez, T. (2011). Improving classification accuracy by identifying and removing instances that should be misclassified. The 2011 International Joint Conference on Neural Networks, 2690-2697. San Jose, CA, USA. doi: 10.1109/IJCNN.2011.6033571.

Tallón-Ballesteros, A. J., \& Riquelme, J. C. (2014). Deleting or keeping outliers for classifier training? Sixth World Congress on Nature and Biologically Inspired Computing (NaBIC 2014), 281-286. Porto, Portugal. doi: 10.1109/NaBIC.2014.6921892.

Turiel, J., \& Aste, T. (2020). Peer-to-peer loan acceptance and default prediction with artificial intelligence. Royal Society Open Science, 7(6). doi:10.1098/rsos.191649.

Turlík, T. (2018). Neural networks and tree-based credit scoring models. Prague: Charles University, Faculty of Social Sciences, Institute of Economic Studies.

Wang, X., Wang, X., \& Wilkes, M. (2021). New Developments in Unsupervised Outlier Detection. Algorithms and Applications. Singapore: Springer. doi: 10.1007/978-981-15-9519-6.

DOI: $10.2478 /$ picbe-2021-0004, pp. 13-32 , ISSN 2558-9652 |

Proceedings of the $15^{\text {th }}$ International Conference on Business Excellence 2021 